\title{
DESIGN AND APPLICATION OF COLLEGE ONLINE EDUCATION PLATFORM BASED ON WEBRTC
}

\author{
GUOLIANG LI $\stackrel{*}{*}$ RIXING WANG † AND QIKUN ZHOU
}

\begin{abstract}
Web based real time communication enhances the current online education platform by blending it with virtual universities by the means of internet. This article explored the designing perspective of College Online Education Platform in order to provide distance education services, instant messaging, interactive online classes, video answering and video viewing functions. A media server cluster load balancing algorithm based on consistency hash algorithm and genetic algorithm is proposed in this article. This article is focused on designing and practical implementation of a fusion communication platform combined with WebRTC and related technologies to deliver online education system in colleges and universities. The online education system is tested and the test results show that the online education system designed and implemented in this paper can meet the expected needs. The media server cluster load balancing strategy proposed in this paper can ensure the cluster overall load balancing. At the same time, the node weight can be dynamically adjusted according to the real-time state of the clusters. The outcomes obtained justifies the efficiency and practicability of the proposed methodology.
\end{abstract}

Key words: WebRTC; University Online Education; Integrated Communication; Load Balancing.

AMS subject classifications. 68M14

1. Introduction. As a unique means of human progress and development, education has deeply influenced and changed people's study and life since ancient times. Traditional conditional education can only be realized by means of paper and pen as media or personal dictation. Nowadays, with the rapid development of computer technology and the continuous improvement of Internet network level, Under the tide of continuous change in many traditional industries, the informatization and networking of education is not only the future direction, but also the urgent need of people. Therefore, a new form of education, that is, online education, or distance education, came into being [1-4]. It is a method of using information technology and Internet technology to spread content and learn quickly.

With the development of Internet technology, books are no longer the only carrier of knowledge transmission, people gradually tend to choose a more convenient way of online education. Although traditional online education can solve the problem of high threshold of students' access to knowledge, due to the lack of effective feedback mechanism, students' access to knowledge is limited. WebRTC technology users do not need to load any plug-in, with cross-platform advantages. Traditional education is a passive mode dominated by teachers, and students' learning initiative is poor [5-8]. And with the development of real-time communication technology, especially the rapid development of WebRTC technology, it will gradually break this one-way way of education, so that students can learn knowledge from anyone at anytime and anywhere $[9,10]$. This can make up for the regional imbalance of education to some extent, so that children in remote areas can also receive quality education $[11,12]$.

This article contributes in the current online education platform by blending it with virtual universities by the means of web based real time communication (WebRTC). It explores the designing perspective of College Online Education Platform for providing the distance education services, instant messaging, interactive online classes, video answering and video viewing functions. The paper proposes a media server cluster load balancing algorithm based on consistency hash algorithm and a genetic algorithm for the implementation of College Online Education Platform Based on WebRTC. The novelty of this article lies in designing and practical

\footnotetext{
*Hunan University of Arts and Science, Changde Hunan, 415000, China (guoliangli1@outlook. com).

${ }^{\dagger}$ Hunan University of Arts and Science, Changde Hunan, 415000, China (rixingwang2@outlook.com).

${ }^{\ddagger}$ Hunan University of Arts and Science, Changde Hunan, 415000, China (qikunzhou74@gmail.com).
} 
implementation of a fusion communication platform combined with WebRTC and related technologies to deliver online education system in colleges and universities. The testing of this online education system is done and the results reveal that the online education system designed and implemented in this paper meet the expected requirements. Comparative to the existing RTC frameworks, the media server cluster load balancing strategy proposed in this article ensures cluster overall load balancing at the same time along with dynamic adjustment to the real-time state of the cluster. The experimental outcomes justify the effectiveness and viability of the proposed methodology.

The rest of this article is structures into different section. Literature review of the current research work going on in this field is presented in section 2. Section 3 presents the system design followed by the results and discussion presented in section 4 . Conclusion of the article is depicted in section 5.

2. Literature Review. Under the current educational situation, many famous educational institutions and educational platforms have emerged at home and abroad. They have different understanding of the forms of network education, and have formed a state of blooming a variety of types. For example, some provide after-class homework and auxiliary videos for auxiliary offline education, some provide communication classes and afterclass evaluation, and some directly set up study groups through chat tools and forums. The emergence of these forms is making the traditional mode of education radiate new charm. Sun, Z. et al. Develop depth learning online intelligent English teaching system, using the creation of a modern tool platform, helping students comply with their knowledge and personality to improve the efficiency of English language teaching. Decision tree algorithm and neural network have been used, and the model is generated based on decision tree technology to generate English teaching assessment. It provides valuable data for extensive information, summarizes rules and data, and help teachers improve their education and English scores. The system reflects the thinking of artificial intelligence expert system. Test applications indicate that systems can help students improve learning efficiency and more relevant learning content. Furthermore, the system provides an example model having a similar method, and has a reference definition [13]. Cheng, L. et al., considered the advanced teaching methods as the research object of cheerleading information curriculum teaching, using literature review methods, questionnaire surveys, interview methods, classroom observation methods and mathematical statistical methods. According to the characteristics of online teaching, the characteristics of cheerleading courses and students' physical/mental characteristics and teaching strategies of cheerlead network courses are designed for providing better education. Cultivation of students' learning habits, learning attitudes, learning skills and thinking innovations, create good environment and learning conditions [14].

The recent advents in the education industry are linked to the diverse benefits which are extensively being used in several universities and colleges for successful operation [15-17]. Kapp [18] concluded that the engagement of students in several games along with teaching may have a positive impact on their mental health and can inculcate the habit of not giving up in the situation of several failures. There are various studies which are evident of improvement in the student's performance when they are involved in gaming as it improves their critical analysis skills while cultivating the problem solving and decision-making qualities of an individual [19, 20]. There has been a focus on games in the present studies which further improves the learning ability $[21,22]$, motivational skills [23], student's attitude for dealing with critical situations [24,25] and inculcate the behavior of participation [26]. There are various positive effects of games on student's personality for e-learning scenario as well. Some of the researchers employed the combination approach including the various data gathering processes for understanding the student's approach for instruction and learning [27]. The mixed strategy involves the gathering of quantitative data from various statistical tests and qualitative information is obtained by student's interview. These approaches are considered useful for developing the traditional e-learning platforms and the student's progress should be monitored from the collected information [28,29].

Based on the research and learning laboratory fusion communication platform and WebRTC technology, this paper will first analyze the requirements of the system, and then determine the overall structure of the online education system in colleges and universities. According to the demand analysis, the whole online education system of colleges and universities mainly includes login verification module, instant messaging module, online classroom module, video answering module and video viewing module [30]. The modules that need to be used WebRTC are online classroom module and video answering module. In order to improve the system concurrency performance, this paper will build multiple media servers into clusters to provide services. 


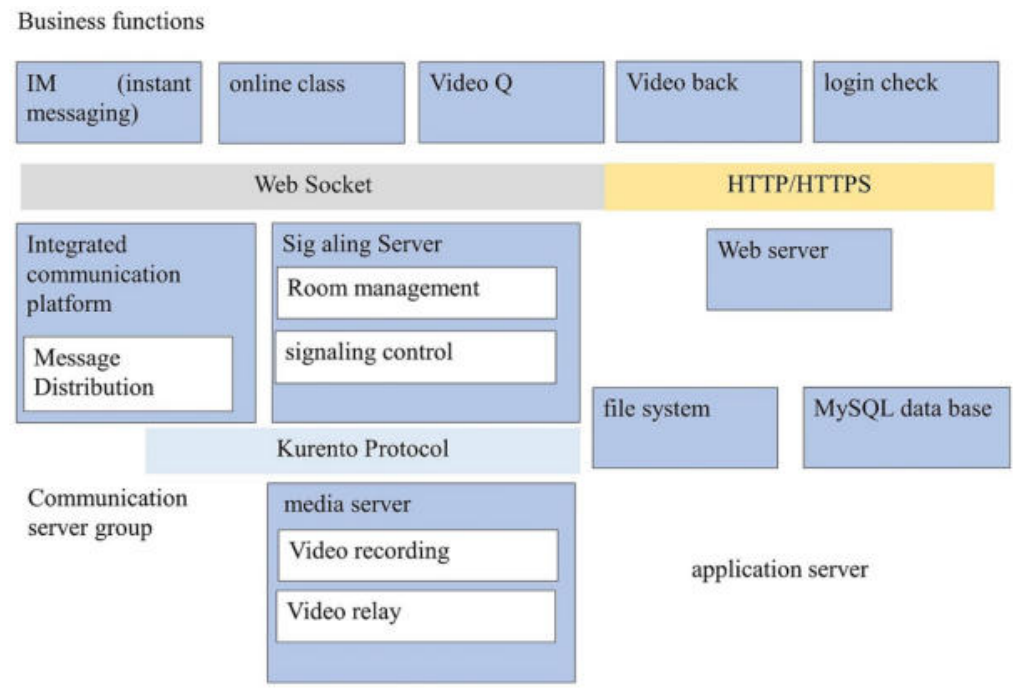

Fig. 3.1. Overall Architecture Map of College Online Education System

\section{System Design.}

3.1. System overall architecture design. The overall architecture diagram of the college online education system (the part related to instant communication and real - time communication) is designed, as shown in Figure 3.1. The top level is the business functions needed to achieve in the college online education system, including login verification module, instant communication module, online classroom module, video question answering module and video back viewing module. The bottom level is the communication server group and application server, which mainly provides communication - related services, such as text, picture expression and real - time audio and video, mainly including integrated communication platform, sign aling server and media server; the application server is mainly used to provide Web services, storage and recording video and provide database services [31]. This paper focuses on the functions related to multimedia communication, that is, the integrated communication platform and WebRTC technology based on laboratory research and development, and designing and implementing the business functions related to text, picture and real-time audio and video communication.

3.2. Design of load balancing algorithm based on consistent $h$ ash and genetic algorithm. In order to meet the consistency demand of the real - time media flow transmission request to the same media server, in this paper, the consistency h ash algorithm is selected as the basic load balancing strategy and optimized using the genetic algorithm.

The basic flow of consistent hash algorithm is: first, the media server node is mapped to a 232-1 loop with a range of values through the hash function, after that, the real-time media streaming request is also mapped to the ring, and the nearest media server node is process the request. However, because there are fewer server nodes, the request may be offset, that is, some server nodes need to process a large number of requests, while other server nodes basically do not process requests, resulting in avalanche effect, which can be solved by using virtual nodes. To a certain extent, the avalanche effect is solved [32].

In the consistency h ash algorithm, the performance differences between the media servers within the cluster can be represented by assigning a different number of virtual nodes to the media server nodes. In the initial state, the number of virtual nodes per media server is often a fixed value of human configuration. In order to ensure the adaptability of the load balancing algorithm, it is necessary to be dynamically adjusted according to the load state of the media server nodes in the cluster, reduce the number of virtual nodes of the media servers with high load, and increase the number of virtual nodes of the media servers with low load. The load rate of 
the media server node can be calculated by Equation (3.1):

$$
L B_{i}=\alpha C_{i}+\beta M_{i}+\gamma B_{i}+\delta L_{i}
$$

where the $L B_{i}$ represents the load rate of the $i$ server node, and the $C_{i}, M_{i}, B_{i}, L_{i}$ are the load balancing index, which represents the CPU utilization, memory utilization, bandwidth utilization ratio, respectively.

$$
\alpha+\beta+\gamma+\delta=1
$$

CPU utilization ratio $C_{i}$ calculated by formula (3.3), $M_{i}$ and $B_{i}$ the same.

$$
C_{i}=C_{t} i / C_{\text {Total }}
$$

The $L_{i}$ number of connections, however, is calculated slightly differently. When a user invites other users in a group to communicate in real time, other users do not necessarily respond immediately, but this part of the connection number also needs to be taken into account. So, multiply a constant $\mathrm{k}$ to indicate the number of connections that will accept invitations, as shown in equation (3.4).

$$
L_{i}=\left(\left(L_{N} i-L_{t} i\right) * k+L_{t} i\right) / L_{\text {Total }}
$$

The media server node regularly sends load information to the load balancer, and then the load balancer calculates the load rate of each media server node $L B_{i}$, When the $L B_{i}>L B_{m} a x$ of the media server node appears, the number of virtual nodes corresponding to each physical server will be recalculated according to formula (3.5) and (3.6), where $V_{t}$ otal, represents the total number of virtual nodes allocated by the cluster.

$$
\begin{gathered}
p_{i}=\left(1-L B_{i}\right) /\left(\sum 1-L B_{i}\right) \\
V_{i}=\left|p_{i} \cdot V_{\text {Total }}\right|
\end{gathered}
$$

After calculating the number of virtual nodes of each physical server, remapping is needed to ensure that all user requests in the group can still be forwarded to the same media server for processing. The information of the media server corresponding to the group needs to be recorded (stored in the Redis database of the signaling server cluster, that is, forwarded directly to the specified media server when the group exists; when it does not exist, the media server is assigned according to the consistency hash algorithm.

However, in the above algorithm, $\alpha, \beta, \gamma, \delta$ the weights of these load balancing parameters are fixed values selected according to experience, they will have a great impact on the results. In order to solve this problem, the weights can be dynamically adjusted by genetic algorithm. Finally, the optimal weights are obtained. The key of using genetic algorithm to solve the problem is to select the appropriate fitness function and genetic operation such as selection, crossover and mutation [33, 34]. Among them, the structure of chromosomes is $\alpha, \beta, \gamma, \delta$ rough empirical value is $(0.2 / 0.25 / 0.35 / 0.2)$, the initial population is 40 , and the value of each position of each chromosome fluctuates not more than 0.05 , which is generated by random algorithm. According to the formulas (3.7), (3.8) and (3.9), the larger the value of the f, the higher the fitness.

$$
\begin{gathered}
\overline{L B}=\left(\sum L B_{i}\right) / N \\
S^{2}=\left(\sum\left(L B_{i}-\overline{L B}\right)^{2}\right) / N \\
f=1 / S
\end{gathered}
$$

The selection operation uses a roulette algorithm to select a chromosome with high adaptivity. At crossoperation, two chromosomes were selected by a random method, and one position on the chromosome was selected by a random method, and then two chromosomes were exchanged in this position, and the probability of crossover operation in this algorithm was $80 \%$. Variation operation, selecting a position of chromosome by a random method, and then floating its up and down does not exceed 0.05 , and the probability of mutation operation in this algorithm is $5 \%$. In order to ensure the stability of the cluster, it does not use the genetic algorithm to change the weight in a certain period of time in the cluster. 




Fig. 3.2. Overall Architecture of Real-time Communication Cluster

3.3. Implementation of Media Server Cluster Load Balancing Strategy. In order to solve the problem of media server cluster load balancing, this paper proposes a load balancing algorithm based on consistency hash algorithm and genetic algorithm [35]. The real-time media flow of clients in the same group is sent to the same media server for processing. The architecture of media server cluster is shown in figure 3.2. The media server node collects the above information every $t$ seconds. It should be noted that the number of bytes sent and the number of bytes received represent the total number of bytes from the beginning to the current moment, so it is necessary to record the last information. Subtracting two data is the number of bytes sent and received in the current $t$ seconds. Media server cluster load balancing algorithm mainly includes two processes. The first process is that the media server node sends the collected CPU utilization, memory utilization, bandwidth utilization and connection ratio to the load balancer every other time. After receiving the above data, the second process is to calculate the load rate of each media server node. When the load rate of the media server node exceeds the system load adjustment threshold, the virtual node will be redistributed for the media server node in the cluster. Each function module of online education system based on WebRTC designed and implemented in this paper is systematically tested to verify whether each module can meet the actual needs. Finally, the performance of the media server cluster load balancing algorithm proposed in this paper is tested.

4. Results and Discussion. The complete system test environment architectural diagram is shown in Figure 4.1.

4.1. System business function test. The login verification module provides users with two login methods: login directly enter the user name password and scan the QR code to login. Users can choose the appropriate login method to login according to the system. The login interface is as shown in Figure 4.1. In the case of client login, users can directly scan the QR code on the left to enter the system, technically the QR code stores a random string (in this system is 8 random digits and letters in the system).

After the user has successfully logged into the system, the user will jump to the system homepage and connect to the MATT agent and the sign align server in the background. Limited to the space constraints, the process for establishing a WebSocket connection is described. To protect it for easy expansion, the WebSocket protocol can connections based on the http (s) protocol. First the client sends a hops request with a " upgrade websocket" head to the sign align server (request address: wss //192.168.139.218/edusys). Then the sign align service returned " 101 Switching Protocols" response means that the client and server have successfully established the WebSocket connection.

For data security, the system uses hops protocol to encrypt the transmitted data, control signaling and 


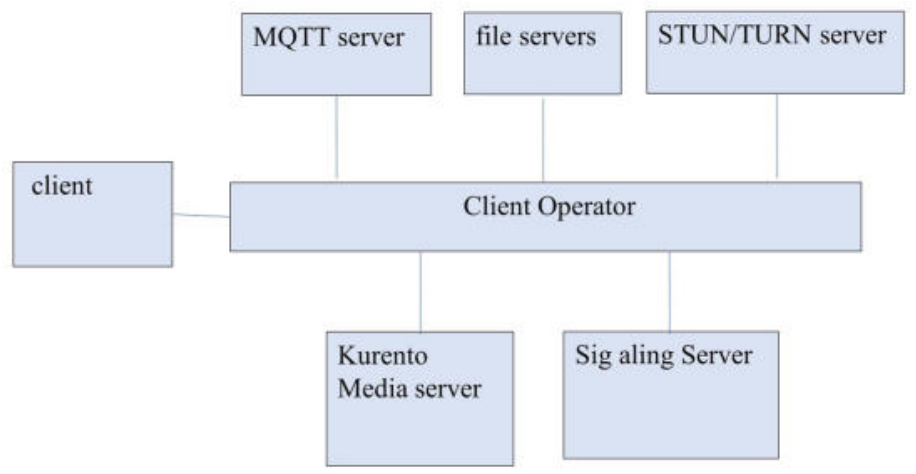

Fig. 4.1. System Test Environment Architecture Map

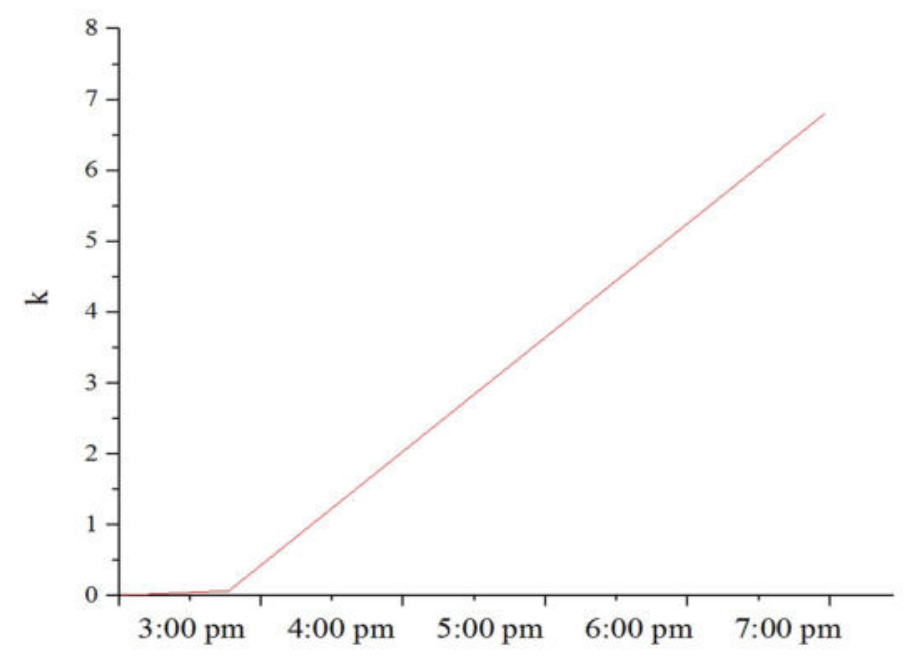

FIG. 4.2. Screen Recording Streaming Data Distribution

real-time media stream. Therefore, the wss connected message captured by the WireShark is encrypted and cannot read any valid data.

The instant messaging module mainly provides users with one-to-one chat and group chat functions. Users can send text, expressions or files in groups. This paper uses Mosquitto server as MATT agent and encrypts instant message by tls protocol to ensure data security. Since the server encrypts the instant message, Wireshark cannot parse the captured tls packet. In order to view the content of the MATT message sent, this paper captures the instant message by Android Studio the breakpoint debugging function. MATT message type is published, message content is "packet capture test". Online classroom module is one of the core functions of the system. Different from the traditional passive learning, while watching the teacher's teaching video, students can ask questions at any time, and the teacher can give answers in class. The whole interface is divided into three parts. The left media stream is the video captured by the teacher's screen, and the upper right media stream is the video collected by the teacher's camera and the audio collected by the microphone. In addition, students can ask questions to teachers through the lower right corner chat box. For ease of testing, Google browser provides the function of viewing WebRTC peer-to-peer connection establishment and sending and receiving monitoring data in the media stream as shown in figure 4.2 , in which the teacher screen captures the data of the video stream.

After the user and the signaling server have established a connection, they will apply to join the room. If the room has not been created, the room will be created, and if it already exists, they will join the room 


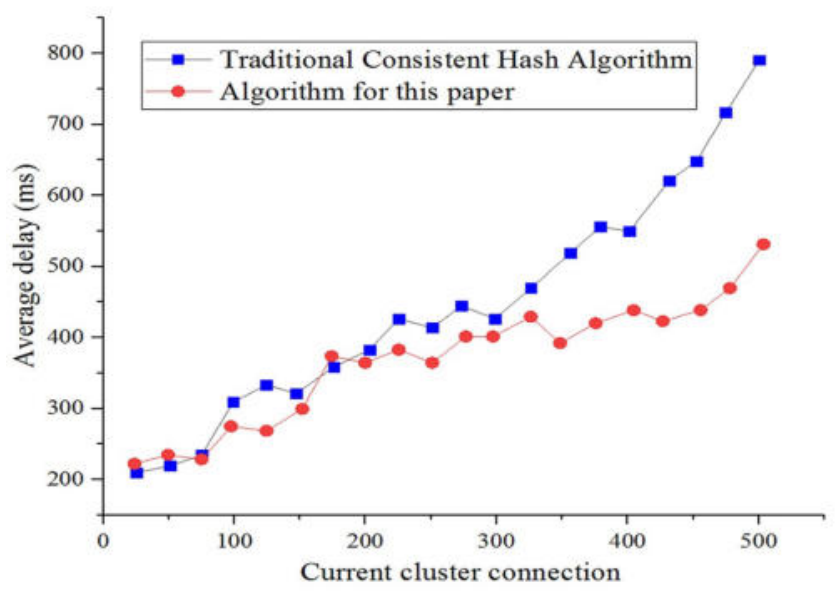

FIG. 4.3. Comparison of the algorithms and traditional consistent hash algorithms in terms of average delay

directly. After the client and the media server establish a connection, after the successful establishment of peer connection, send the user's media stream, if there are other users in the room, then receive the real-time media stream of other users. When a user joins the room, he receives the user's real-time media stream; similarly, when a user leaves the room, he stops receiving the user's media stream.

This module provides users with the function of watching back teaching video, that is, recording real-time audio and video released by teachers in online classroom module for students to watch. If the course has been recorded or recorded, the user can click the "look back video" button to watch the video.

4.2. Performance Test of Media Server Cluster Load Balancing Strategy. This paper uses the modified NUBOMEDIA benchmark tool to test the media server load balancing algorithm proposed in this paper, and compares the test results with the traditional consistent Hash algorithm, in which the video stream is coded by H.264, the frame rate is $30 \mathrm{fps}$, the resolution is $600 \mathrm{x} 800$; the audio stream is coded by Opus, and the sampling rate is $48 \mathrm{kHz}$. In order to facilitate the test, the client connects to the media server and continues to open the connection. The experiment is carried out in the LAN environment.

When the number of cluster connections is small, the average delay between this algorithm and the traditional consistent hash algorithm is not different, but with the increase of the number of connections, the average delay of this algorithm is shorter. As shown in figure 4.3, the average delay can reflect the efficiency of load balancing to some extent, but it is not accurate to use the average delay to evaluate the load balancing efficiency of the algorithm. To measure the load balancing efficiency of the algorithm more accurately, as shown in figure 4.4. It can be seen that the variance of load rate of this algorithm is relatively low, that is, the algorithm can balance cluster load more than the traditional consistent hash algorithm.

As the client connects to the server, the load rate of each server will continue to rise, but the overall load of each server in the cluster is balanced. Figure 4.5 is representative of the cluster load balancing results in terms of server load rate; however, the server connection comparison with the current cluster connection is done in Figure 4.6. Although the server load is balanced, the server allocation is different because of server performance difference.

Through the test of the whole function of the page, each module runs well in function and is consistent with the expected results. Through the compatibility test of the system, the actual support of the system audio and video module is consistent with the theory. Overall, the system in all aspects of good performance, to meet the expected needs.

5. Conclusion. A WebRTC-based distance education system is designed and implemented in this paper. The experimental tests done reveals that the designing and practical implementation of a fusion communication platform combined with WebRTC meet the expected requirements. This article proposes a media server cluster load balancing strategy which ensures the cluster overall load balancing while dynamically adjusting the node 


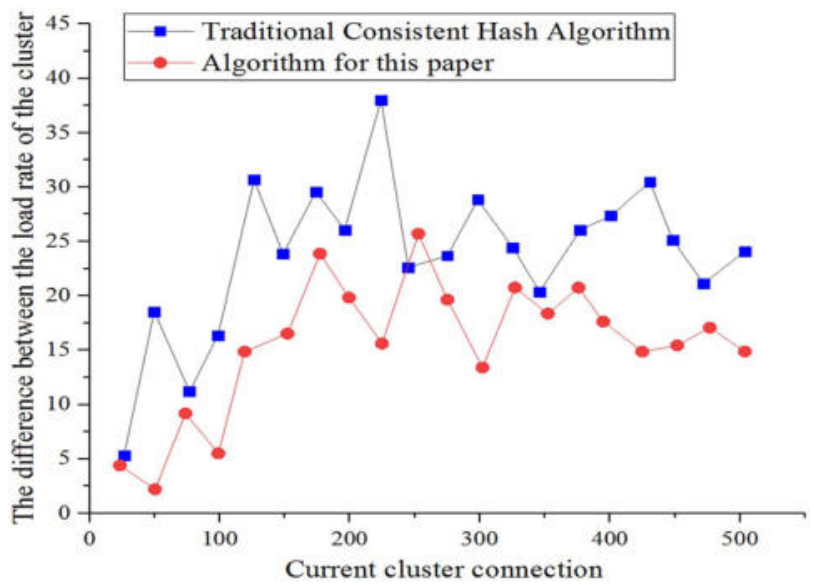

FIG. 4.4. Comparison of the algorithms and traditional consistent hash algorithms in terms of difference between the load rate of clusters

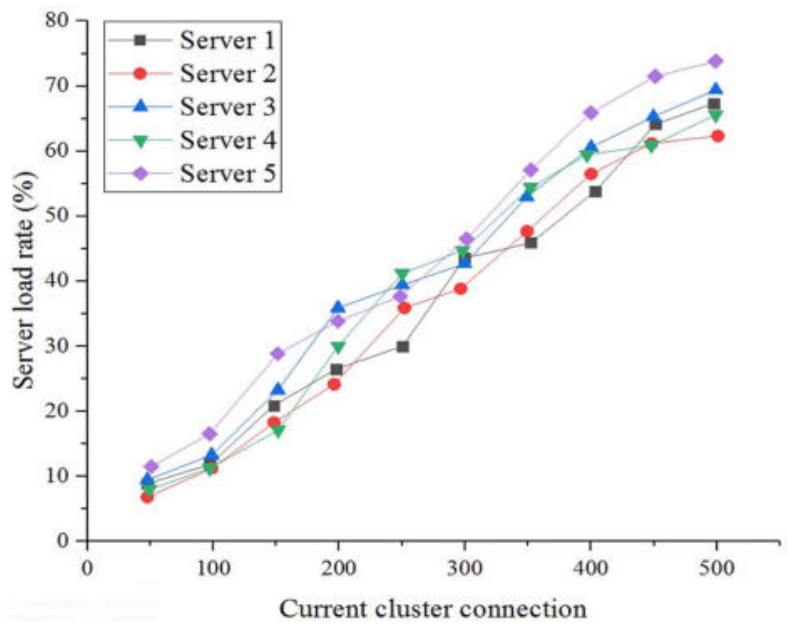

FIG. 4.5. The results of cluster load balancing in terms of server load rate

weights as per the real-time state of the clusters. The experimental outcomes justify the effectiveness and viability of the proposed methodology. There are some future scopes for improvement which can be studied and optimized in the future.

1. The system in this paper can achieve the desired goal, but it needs to further optimize and improve the system function according to the user feedback later.

2. The proposed load balancing strategy of media server cluster can achieve good experimental results, but the research on how to adjust the load of the system to the equilibrium state quickly is still required, therefore, further optimization can be done as a future perspective. Also, the future research directions of this work will consider Wi-Fi communication environment for experimentation analysis.

Acknowledgments. 1. Research project of teaching reform in universities of Hunan province (Item no.HNJG-2020-0728) Project Leader: Guoliang Li.

2. Research project of Teaching Reform Research Project of Hunan Universities (Item no.- HNJG-20200727) Project Leader: Rixing Wang. 


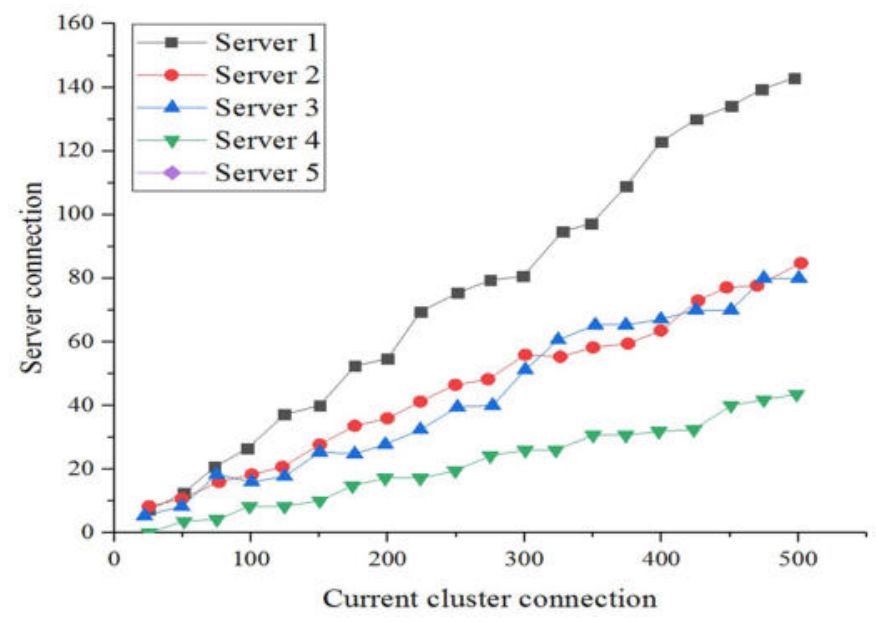

FIG. 4.6. The results of cluster load balancing comparing the server connection and current cluster connections

\section{REFERENCES}

[1] Han, C. , Design and Application of the Intelligent Learning Platform for College English Based on the New Engineering Concept, In International Conference on Application of Intelligent Systems in Multi-modal Information Analytics, 60-66, 2019.

[2] Watanabe, T., A WebRTC e-Learning System Based on Kurento Media Server, In E-Learning and Games: 12th International Conference, Edutainment 2018, Xi'an, China, 11462,331, 2019.

[3] Pakinee, A., AND PuRITAT, K., Designing a gamified e-learning environment for teaching undergraduate ERP course based on big five personality traits, Education and Information Technologies, 1-19, 2021.

[4] Gu, X., Yang, L., Liang, Y., And CaO, S., Design and implementation of digital assets management system based on WeChat, In 2017 IEEE 2nd Information Technology, Networking, Electronic and Automation Control Conference (ITNEC), 394-398, 2017.

[5] He, C., Fu, Z., Liu, H., AND LI, G., Development of mobile learning system based on WeChat public platform, In 2019 10th International Conference on Information Technology in Medicine and Education (ITME), 363-367, 2019.

[6] Schwade, F., AND SchuberT, P., The ERP challenge: an integrated e-learning platform for the teaching of practical ERP skills in universities, Procedia Computer Science, 100, 147-155, 2016.

[7] Kattoua, T., Al-Lozi, M., And Alrowwad, A. A., A review of literature on E-learning systems in higher education, International Journal of Business Management and Economic Research, 7(5), 754-762, 2016.

[8] Coman, C., Țîru, L. G., Meseșan-Schmitz, L., Stanciu, C., And Bularca, M. C., Online Teaching and Learning in Higher Education during the Coronavirus Pandemic: Students' Perspective,Sustainability, 12(24), $10367,2020$.

[9] Popa, D., Repanovici, A., Lupu, D., Norel, M., And Coman, C., Using Mixed Methods to Understand Teaching and Learning in COVID 19 Times, Sustainability, 12(20), 8726, 2020.

[10] Burac, M. A. P., Fernandez, J. M., Cruz, M. M. A., And Cruz, J. D., Assessing the impact of e-learning system of higher education institution's instructors and students, In IOP Conference Series: Materials Science and Engineering, 482(1), 012009, 2019.

[11] Stott, A., And Neustaedter, C., Analysis of gamification in education, Surrey, BC, Canada, 8, $36,2013$.

[12] De-Marcos, L., Domínguez, A., SAenz-De-Navarrete, J., And Pagés, C., An empirical study comparing gamification and social networking on e-learning, Computers and education, 75, 82-91, 2014.

[13] Sun, Z., Anbarasan, M., And Kumar, D. P., Design of online intelligent english teaching platform based on artificial intelligence techniques, Computational Intelligence, 2020.

[14] Cheng, L., Niu, W. C., Zhao, X. G., Xu, C. L., And Hou, Z. Y., Design and implementation of college physics teaching platform based on virtual experiment scene, International Journal of Electrical Engineering Education, 002072092098468, 2021.

[15] Urh, M., Vukovic, G., AND Jereb, E., The model for introduction of gamification into e-learning in higher education, Procedia-Social and Behavioral Sciences, 197, 388-397, 2015.

[16] Chang, J. W., AND WEI, H. Y., TExploring engaging gamification mechanics in massive online open courses, Journal of Educational Technology and Society, 19(2), 177-203, 2016.

[17] Hussein, M. H., Ow, S. H., Cheong, L. S., And Thong, M. K., A digital game-based learning method to improve students' critical thinking skills in elementary science, IEEE Access, 7, 96309-96318, 2019.

[18] KAPP, K. M., The gamification of learning and instruction: game-based methods and strategies for training and education, John Wiley and Sons, 2012.

[19] Attali, Y., AND Arieli-Attali, M., Gamification in assessment: Do points affect test performance?, Computers and 
Education, 83, 57-63, 2015.

[20] Gibson, L., And Hanson, V. L., Digital motherhood: How does technology help new mothers?, In Proceedings of the SIGCHI conference on human factors in computing systems, 313-322, 2013.

[21] De-Marcos, L., Domínguez, A., Saenz-De-Navarrete, J., And Pagés, C., An empirical study comparing gamification and social networking on e-learning, Computers and education, 75, 82-91, 2014.

[22] Glover, I., Play as you learn: gamification as a technique for motivating learners, In Edmedia+ innovate learning, 1999-2008, 2013.

[23] Monu, K., And Ralph, P., Beyond gamification: implications of purposeful games for the information systems discipline, arXiv preprint arXiv:1308.1042, 2013.

[24] Alcivar, I., And Abad, A. G., Design and evaluation of a gamified system for ERP training, Computers in Human Behavior, $58,109-118,2016$.

[25] Chen, C. L. D., Yen, T. K., And Chang, C. Y., The effects of game-based learning and anticipation of a test on the learning outcomes of 10th grade geology students, Eurasia Journal of Mathematics, Science and Technology Education, 12(5), 1379-1388, 2016.

[26] , L., Er, E., and Orey, M., An exploratory study of student engagement in gamified online discussions, Computers and Education, 120, 213-226, 2018.

[27] Lo, C. K., AND Hew, K. F., A comparison of flipped learning with gamification, traditional learning, and online independent study: the effects on students' mathematics achievement and cognitive engagement, Interactive Learning Environments, $28(4), 464-481,2020$.

[28] Zainuddin, Z., Shujahat, M., Haruna, H., and Chu, S. K. W., The role of gamified e-quizzes on student learning and engagement: An interactive gamification solution for a formative assessment system, Computers and Education, 145, 103729, 2020.

[29] Sanchez, D. R., Langer, M., And Kaur, R., Gamification in the classroom: Examining the impact of gamified quizzes on student learning,Computers and Education, 144, 103666, 2020.

[30] Zhang, X., Research on the design of college english lessons based on hybrid-styled teaching,Journal of Contemporary Educational Research, 4(1), 2020.

[31] YanG, X., AND Song, Z., College students' law education platform based on browser/server and massive open online courses, International Journal of Emerging Technologies in Learning (iJET), 14(15), 202, 2019.

[32] Lin, W., Study on the design and application of the user information resources of track and field web course based on software programming method, ICluster Computing, 22(15), 1-9, 2019.

[33] LI, M., LI, Y., AND GuO, H., Research and application of situated teaching design for NC machining course based on virtual simulation technology,Computer Applications in Engineering Education, 28(3), 658-674, 2020.

[34] Yan, Y., Design and Implementation of a Teaching Assistance Platform for College Students Based on ASP. NET, International Journal of Emerging Technologies in Learning, 14(12), 2019.

[35] Pireddu, G., Svir, I., Amatore, C., And Oleinick, A., Interactive competition between individual diffusion layers during cyclic voltammetry at random arrays of band and disk electrodes: a thorough analysis based on global simulations, ChemElectroChem, 2021.

Edited by: Pradeep Kumar Singh

Received: May 16, 2021

Accepted: Sep 20, 2021 